\title{
Use of the Control of Allergic Rhinitis and Asthma Test and pulmonary function tests to assess asthma control in pregnancy.
}

Amaral $\mathrm{L}^{1}, \underline{\text { Martins } \mathrm{C}^{1}}, \underline{\text { Coimbra } \mathrm{A}^{1}}$.

\section{Abstract}

\section{Introduction}

Asthma is one of the most prevalent chronic medical conditions to complicate pregnancy. Similarly, active management strategies that prioritise asthma control in this vulnerable population can have a far-reaching impact.

\section{Aim}

To describe lung function parameters and results of the Control of Allergic Rhinitis and Asthma Test (CARAT) questionnaire in pregnant asthmatics and to analyse correlations between lung function tests and CARAT results.

\section{Methods}

All pregnant asthmatics who performed spirometry and CARAT between September 2014 and August 2015 were included. A medical records review was performed and data regarding demographics, sensitisation and medical prescriptions were recorded. Control of asthma and rhinitis was defined by CARAT total score (CARAT $(\mathrm{T})) \geq 24$; upper airways $(\mathrm{U})$ control if CARAT $(\mathrm{U})>8$; lower airways $(\mathrm{L})$ control if CARAT (L) $>15$.

\section{Results}

Forty-two pregnant asthmatics were included, with a median age (interquartile range)of 32 (27-34) years; all had a previous medical diagnosis of asthma and rhinitis, $76 \%$ were atopic, $94 \%$ sensitised to dust mites and $43 \%$ were polissensitised. More than $80 \%$ presented poor control (CARAT $(\mathrm{T})<24$ ) in the first assessment and $15 \%$ had a percentage forced expiratory volume in the first second $<80 \%$. There were significant negative correlations between CARAT (L) and CARAT (T) scores and step-up of controller medication, (correlation coefficients $=-0.453$ and -0.392 , respectively).

\section{Conclusion}

The use of tools such as spirometry and validated questionnaires to objectively assess asthma control during pregnancy should be routinely applied to assist in the 
management of these patients. These data reinforce the importance of close monitoring of pregnant asthmatics to achieve and maintain better disease control.

\section{Introduction}

Asthma is one of the most common chronic diseases of women of childbearing age, 1 complicating up to $8 \%$ of pregnancies in the United States 2 and 12\% in Australia.3

The course of asthma during pregnancy is unpredictable. Generally, asthma worsens during pregnancy in approximately one-third of women, one-third improves and the other third remains unchanged. 4

Maternal asthma is associated with a higher rate of adverse perinatal outcomes, including intrauterine growth restriction (IUGR), preterm delivery and preeclampsia.5, 6 Moreover, asthma exacerbations are associated with an increased risk of any congenital malformation. 7 On the other hand, when properly controlled, asthma is associated with a favourable fetal and maternal prognosis and well-controlled asthma is fundamental for an uncomplicated pregnancy and a healthy newborn. 3 A subjective assessment of asthma control has little benefit in the discrimination of controlled and uncontrolled patients and this may lead to insufficient treatment or excessive use of medication. 8

In pregnant women, shortness of breath is the most common respiratory complaint. Nonetheless, this is not associated with other typical asthma symptoms such as wheezing, chest tightness and cough.9 Thus, asthma control evaluation in pregnant asthmatics, may be a challenge for clinicians because of the many physiological peculiarities associated with the pregnancy itself.

According to the Global Initiative for Asthma (GINA), control is based on asthma symptoms, limitation on daily life activities, nocturnal symptoms/awakenings, exacerbations with the need of rescue medication and lung function tests (forced expiratory volume in the first second (FEV1)). 10

In order to achieve and maintain asthma control in pregnancy, regular assessment and appropriate treatment of asthma and concomitant diseases that 
can affect the control is necessary. Allergic rhinitis (AR) is frequently associated with asthma and its control. According to the Allergic Rhinitis and its Impact on Asthma (ARIA) recommendations, the management of these two conditions must be held jointly.11, 12 However, the tools available to objectively evaluate AR control are scarce.

The Control of Allergic Rhinitis and Asthma Test (CARAT) is the only validated self-administered questionnaire that evaluates asthma and AR control concurrently and it was developed in Portugal.13 The first study that assessed the association between the results of the CARAT questionnaire and spirometry parameters, performed in adults with asthma and/or AR, demonstrated a weak correlation between CARAT scores and percentage of predicted FEV1 (\%FEV1).14 To our knowledge, no study has evaluated the association of CARAT scores and spirometry in pregnant asthmatics.

The primary aim of this study was to describe the lung function parameters and the results of the CARAT questionnaire in a series of pregnant women with asthma and/or AR. As a secondary objective, we sought to analyse possible associations between CARAT scores and spirometric data during pregnancy.

\section{Materials and Methods}

This was a longitudinal study, including pregnant women with asthma and AR followed in an Allergy and Clinical Immunology Department.

Pregnant asthmatics were selected based on the data of lung function tests carried out between September 2014 and August 2015. During this period, all pregnant women who underwent spirometry, were invited to complete the CARAT. Inhaler technique was assessed at each evaluation. Lung function parameters (\%FEV1), spirometry classification, demographic and anthropometric characteristics (age, body mass index (BMI)) and CARAT results were collected. A medical records review was performed and clinical data regarding clinical diagnosis, atopic sensitisation, smoking habits, acid reflux, need of rescue and step up of control medication were recorded. The CARAT is a self-administered questionnaire assessing asthma and AR control 
in the last 4 weeks. It is composed of 10 questions divided into two scores: score 1 (CARAT (U)), made up of four questions regarding symptoms of the upper airways and score 2 (CARAT (L)), including four questions specifically related to lower airways symptoms, one sleep-related symptoms and one with additional medication (evaluated jointly).13 The question regarding medication had three response options ('Never' - three points, 'Less than seven days' - two points 'More than seven days' - 0 points) and an option 'I'm not taking any medication' which was also attributed three points. All the questions are scored on a Likert scale of four points and the total score CARAT (T) can vary between 0 and 30 points, corresponding to the worst and best possible control, respectively. The partial scores score 1 CARAT (U) of more than eight points, the score 2 CARAT (L) greater than 15 points and the total score CARAT (T) greater than 24, are indicative of good control.

Spirometry was performed according to the European Respiratory Society/American Thoracic Society (ERS/ATS) guidelines 15 using a Jaeger spirometer, MasterScope (VIASYS Healthcare, Hoechberg, Germany). Spirometry was classified as normal, small airway obstruction (with normal FEV1), restrictive ventilatory defects, mixed ventilatory defects and mild obstructive (FEV1 $\geq 70 \%$ ), moderate $(50 \% \leq \mathrm{FEV} 1<70 \%)$ and severe (FEV1 $<50 \%$ ). Classification of obstructive ventilatory defects was based on the FEV1and the forced vital capacity ratio (FEV1/FVC), and a ratio FEV1/FVC $<70 \%$ indicates airway obstruction.

\section{Study population}

All pregnant women who underwent spirometry during the study period were included. Those with only one lung function assessment were excluded (Fig. 1). 


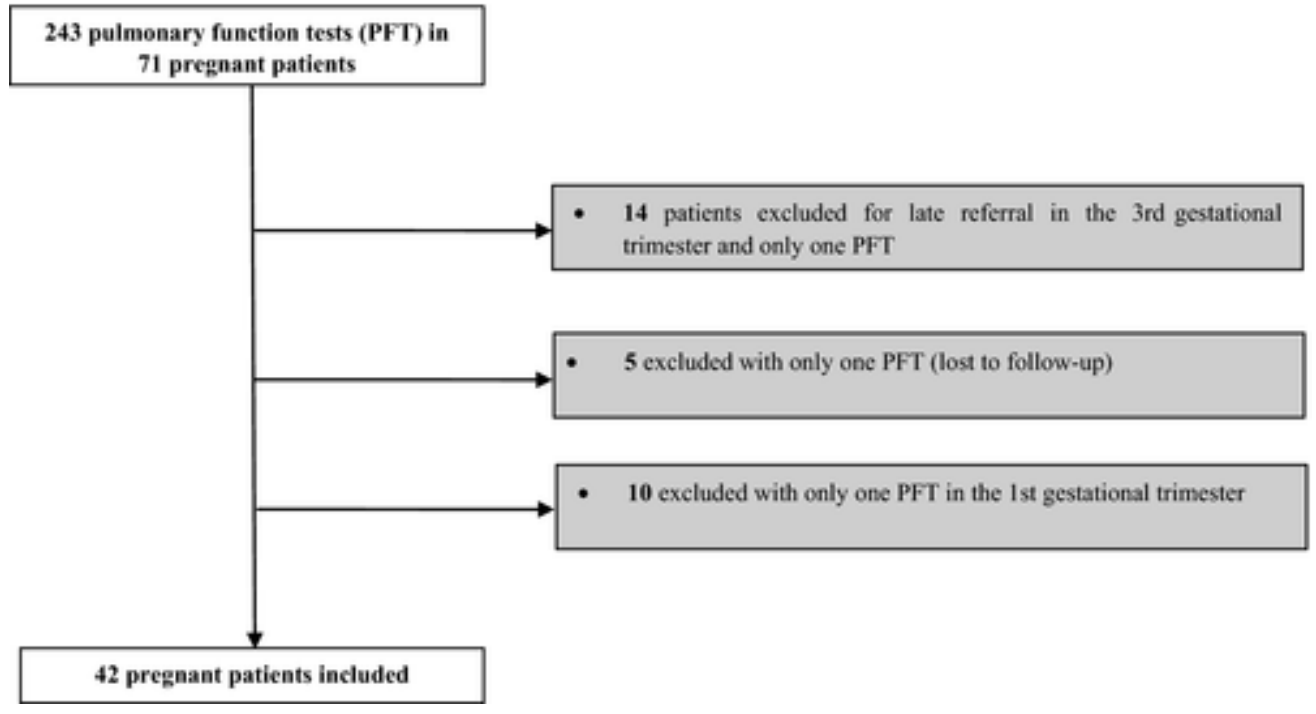

\section{Figure 1}

Open in figure viewerPowerPoint

Participant selection flowchart.

\section{Statistical analysis}

Statistical analysis was performed using SPSS version 22 (IBM, Armonk, NY, USA). Appropriate descriptive measures were used, including mean and standard deviation for continuous variables with normal distribution and median and interquartile ranges (IQR) for continuous variables with non-normal distribution. The $\chi^{2}$ test was used for comparing proportions and Student's- $t$ test in comparison of means in independent samples.

The correlations between the results of CARAT, \%FEV1 and the need for therapeutic step-up were calculated using Spearman rank correlation coefficient. A $P=$ value below 0.05 was considered statistically significant.

\section{Ethical approval}

The study was approved by the local ethics committee, Ethics Committee Centro Hospitalar São João/Faculty of Medicine, University of Porto. (Ref. no CES 47-15, date of approval: 26 March 2015).

\section{Results}

Forty-two pregnant women were included (Fig. 1), all with a previous medical diagnosis of asthma and rhinitis. The median age (IQR) was 32 (27-34) years. All were non-smokers. Thirty-two (76\%) were atopic, 30 (94\%) sensitised to 
dust mites, $16(50 \%)$ to pollens, $9(28 \%)$ cat and/or dog dander and $18(43 \%)$ were polysensitised (Table 1).

Table 1. Patients' clinical characteristics

Age, median (IQR), years

Body mass index at first assessment

Underweight $(<20)$

Normal range (20-25)

$13(31)$

Overweight (25-30)

Obesity $(>30)$

$10(24)$

Diagnosis

Asthma and rhinitis

$42(100)$

Atopic dermatitis

$4(10)$

Urticaria 
Treatment (first assessment)

No treatment

$14(33)$

Inhaled corticosteroids 
- IQR, interquartile range.

- Data are presented as $n(\%)$, unless stated otherwise.

At the first observation, $10(24 \%)$ were obese (BMI > 30). Fourteen (33\%) did not have any controller medication for asthma and rhinitis, 27 (64\%) used inhaled corticosteroids, six (14\%) used topical nasal steroids and 31 (74\%) were medicated with antihistamines for exacerbations (Table 1).

Of the 14 women without controller medication, $64 \%$ reported that they were not on any medication because of fear of harm to the fetus and $36 \%$ were advised to interrupt their medication by the doctor who was monitoring the pregnancy.

In the first evaluation, almost $15 \%$ of the pregnant patients had $\% \mathrm{FEV} 1<80 \%$ and approximately $30 \%$ had an abnormal spirometry. In these, almost $17 \%$ presented moderate obstruction and none had severe obstruction or mixed/restrictive ventilatory defects. The spirometry classification, performed throughout the different assessments during the pregnancy remained constant (Table 2).

Table 2. Pulmonary function tests (PFT) and control during pregnancy 


\begin{tabular}{|c|c|c|c|}
\hline & $\begin{array}{l}\text { First assessment } \\
\qquad(n=42)\end{array}$ & $\begin{array}{c}\text { Second } \\
\text { Assessment } \\
(n=42)\end{array}$ & $\begin{array}{c}\text { Third } \\
\text { Assessment } \\
(n=22)\end{array}$ \\
\hline $\begin{array}{l}\text { Gestational age (weeks), } \\
\text { median (IQR) }\end{array}$ & $18(13-24)$ & $28(22-33)$ & $29(25-35)$ \\
\hline$\%$ FEV1, mean (SD) & $97,2(13,4)$ & $98,9(13,4)$ & $97,3(16,1)$ \\
\hline PFT classification & & & \\
\hline Normal & $30(71,4)$ & $29(69)$ & $13(59,1)$ \\
\hline Mild obstruction & $9(21,4)$ & $10(23,8)$ & $7(31,8)$ \\
\hline Moderate obstruction & $2(4,8)$ & $1(2,4)$ & - \\
\hline $\begin{array}{l}\text { Small airway } \\
\text { obstruction }\end{array}$ & $1(2,4)$ & $2(4,8)$ & $2(9,1)$ \\
\hline CARAT (U) & & & \\
\hline Score, mean (SD) & $5,8(3,2)$ & $7,2(2,9)$ & $7,6(3,3)$ \\
\hline Uncontrolled & $31(74)$ & $24(57,1)$ & $10(45,5)$ \\
\hline
\end{tabular}




\begin{tabular}{|c|c|c|c|}
\hline & $\begin{array}{l}\text { First assessment } \\
\qquad(n=42)\end{array}$ & $\begin{array}{c}\text { Second } \\
\text { Assessment } \\
(n=42)\end{array}$ & $\begin{array}{c}\text { Third } \\
\text { Assessment } \\
(n=22)\end{array}$ \\
\hline CARAT (L) & & & \\
\hline Score, mean (SD) & $11,9(4,9)$ & $13,4(4,6)$ & $14,4(4,1)$ \\
\hline Uncontrolled & $30(71)$ & $23(54,8)$ & $12(54,5)$ \\
\hline CARAT (T) & & & \\
\hline Score, mean (SD) & $17,7(7,4)$ & $20,6(6,3)$ & $22,0(7,0)$ \\
\hline Uncontrolled & $34(81,0)$ & $30(71,4)$ & $11(50,0)$ \\
\hline Step up medication & - & $4(9,5)$ & - \\
\hline
\end{tabular}

- CARAT (L), Control of Allergic Rhinitis and Asthma Test (lower airways); CARAT (T), CARAT (total); CARAT (U), CARAT (upper airways); IQR, interquartile range; $\% \mathrm{FEV} 1$, percentage of forced expiratory volume in the first second; SD, standard deviation.

- Data are presented as $n(\%)$, unless stated otherwise.

At the first visit, the assessment of the upper airway symptoms using CARAT (U) score showed poor control in $74 \%$ and the scores of the lower respiratory tract symptoms according to CARAT (L) also showed poor control in $71 \%$. A 
significant improvement in the second evaluation was observed $(P=0.01)$. Correlations between the CARAT scores and \%FEV1 results were not statistically significant. On the other hand, significant negative correlations were found between the scores of CARAT (L) and CARAT (T) and the need for stepping up controller medication (correlation coefficient $(C C)=-0.453, P=0.003)$ and $C C=-0.392 ; P=0.01$, respectively). None of the pregnant asthmatics required oral corticosteroids for exacerbations.

Eighteen pregnant women (43\%) complained of having an acid taste in the mouth and regurgitation at least 1 day per week, particularly in the third trimester. All reported controlling these symptoms with dietary and lifestyle changes without pharmacological treatment. Further comparison revealed no significant differences between acid-reflux symptoms and asthma exacerbations $(P=0.73)$ or step-up controller medication $(P=0.61)$.

\section{Discussion}

The goal of asthma treatment in pregnant women is to achieve control in order to prevent exacerbations, maintain maternal health and optimise fetal growth. Treatment should be adjusted to the lowest dose to maintain control.

Despite the importance of adequate treatment 16 and its safety, there is still interruption of treatment by pregnant women or by the clinicians who followed the pregnancies. In this series, nearly two-thirds of the 14 pregnant asthmatics without medication reported fear of harm to the fetus as the reason for noncompliance and the remaining patients stopped treatment because of advice given by their attending physicians.

Acid-reflux symptoms are very frequent in pregnant women and they increase in severity during pregnancy. The pathophysiologic mechanisms involved in abnormal gastric reflux during pregnancy are decreased lower oesophageal sphincter pressure and alterations in gastrointestinal transit due to hormone changes, and increased intra-abdominal pressure secondary to the enlarged gravid uterus. Asthma and gastro-oesophageal reflux disease (GERD) are common comorbidities and the latest evidence-based guidelines (GINA 2017) recommend that GERD should be treated in order to help reduce issues with 
asthma. However, published studies so far have not been conclusive as to the impact of treatment. There may be a connection between controlling GERD to aid in asthma control but high-quality research to prove this is lacking. More robust research is required to establish or remove this link.10 Moreover, in this sample, acid reflux symptoms were not associated with asthma exacerbations and the need for step-up of asthma medication.

Allergic rhinitis is frequently associated with asthma and it may require the use of topical nasal corticosteroids and/or antihistamines. In this study, only $14 \%$ of the pregnant women were medicated with nasal corticosteroids but $74 \%$ resorted to antihistamines.

Asthma in pregnancy should be monitored more regularly and this should include lung function evaluation, as recommended by the guidelines. 3 In this sample, the mean \%FEV1 throughout the evaluations remained above $80 \%$. However, in the first evaluation, nearly $30 \%$ of the pregnant women had ventilatory obstructive defects, but none with severe obstruction or mixed/restrictive changes. This distribution of obstructive changes remained constant throughout the different assessments.

In relation to CARAT (T), there was poor control in the first evaluation in almost $81 \%$ of the pregnant women, as well as CARAT (U) in $74 \%$ and CARAT (L) in $71 \%$ of the patients. There was a significant improvement in control for the three scores throughout the different assessments. These results may be related to better adherence to treatment and closer medical supervision.

No significant correlation was found between CARAT scores and \%FEV1. This correlation was weak in previous studies with CARAT14 and other asthma control questionnaires.17, 18 This may be explained by the sample size.

However, significant negative correlations were detected between the CARAT (T) and CARAT (L) scores and the need for stepping up controller medication. 
In our opinion, these results support the combined use of CARAT and lung function tests in the clinical assessment of asthma during pregnancy, for closer monitoring and the evaluation of any necessary treatment adjustments.

\section{Conclusion}

The joint use of tools such as spirometry and validated questionnaires, particularly the CARAT, to objectively assess asthma control during pregnancy should be applied on a regular basis. The data presented in this study reinforces the importance of close monitoring of pregnant asthmatics in order to achieve and maintain control of the disease.

\section{- References}

1Belanger K, Hellenbrand ME, Holford TR, Bracken M. Effect of pregnancy on maternal asthma symptoms and medication use. Obstet Gynecol 2010; 115(3): 559-567.

- 2Kwon HL, Belanger K, Bracken MB. Asthma prevalence among pregnant and childbearing-aged women in the United States: estimates from national health surveys. Ann Epidemiol 2003; 13(5): 317-324.

- 3Vanders RL, Murphy VE. Maternal complications and the management of asthma in pregnancy. Women's Health 2015; 11(2): 183-191.

- 4Schatz M, Dombrowski MP, Wise R et al. Asthma morbidity during pregnancy can be predicted by severity classification. J Allergy Clin Immunol 2003; 112(2): 283288.

- 5Dombrowski MP, Schatz M. Asthma in pregnancy. Clin Obstet Gynecol 2010; 53(2): 301-310.

- 6Murphy V, Namazy J, Powell H et al. A meta-analysis of adverse perinatal outcomes in women with asthma. BJOG 2011; 118(11): 1314-1323.

- 7Blais L, Forget A. Asthma exacerbations during the first trimester of pregnancy and the risk of congenital malformations among asthmatic women. $J$ Allergy Clin Immunol 2008; 121(6): 1379-1384. e1371.

- 8Accordini S, Bugiani M, Arossa W et al. Poor control increases the economic cost of asthma. Int Arch Allergy Immunol 2006; 141(2): 189-198.

- 9Bidad K, Heidarnazhad H, Pourpak Z et al. Frequency of asthma as the cause of dyspnea in pregnancy. Int J Gynecol Obstet 2010; 111(2): 140-143.

- 10Global Strategy For Asthma Management And Prevention (updated 2017): Global Initiative for Asthma (GINA). [Accessed 26 April 2017.] Available from URL: http://www.ginasthma.org. 
- 11Brożek JL, Bousquet J, Baena-Cagnani CE et al. Allergic Rhinitis and its Impact on Asthma (ARIA) guidelines: 2010 revision. J Allergy Clin Immunol 2010; 126(3): 466-476.

- 12Bousquet J, Schünemann H, Zuberbier T et al. Development and implementation of guidelines in allergic rhinitis-an ARIA-GA2LEN paper. Allergy 2010; 65(10): 1212-1221.

- 13Fonseca J, Nogueira-Silva L, Morais-Almeida M et al. Validation of a questionnaire (CARAT10) to assess rhinitis and asthma in patients with asthma. Allergy 2010; 65(8): 1042-1048.

- 14Pereira A, Martins C, Fonseca J. Use of CARAT and lung function tests to assess control of asthma and rhinitis. Rev Port Imunoalergologia 2013; 21: 103115.

- 15Miller MR, Hankinson J, Brusasco V et al. Standardisation of spirometry. Eur Respir J 2005; 26(2): 319-338.

- 16Maselli DJ, Adams SG, Peters JI, Levine SM. Management of asthma during pregnancy. Ther Adv Respir Dis 2013; 7(2): 87-100.

- $\quad$ 17Melosini L, Dente FL, Bacci E et al. Asthma control test (ACT): comparison with clinical, functional, and biological markers of asthma control. J Asthma 2012; 49(3): 317-323.

- 18Park SY, Yoon S-Y, Shin B et al. Clinical factors affecting discrepant correlation between asthma control test score and pulmonary function. Allergy Asthma Immunol Res 2015; 7(1): 83-87. 